\title{
Characterization of the tufB-secE-nusG-rplKAJL-rpoB Gene Cluster of the Citrus Greening Organism and Detection by Loop-Mediated Isothermal Amplification
}

\author{
Mitsuru Okuda, Mitsuhito Matsumoto, and Yuko Tanaka, National Agricultural Research Center for Kyushu \\ Okinawa Region, Suya 2421, Nishigoshi, Kumamoto 861-1192, Japan; Siti Subandiyah, Department of Entomology \\ and Plant Pathology, Gadjah Mada University, Yogyakata 55281, Indonesia; and Toru Iwanami, National Agricul- \\ tural Research Center for Kyushu Okinawa Region, Suya 2421, Nishigoshi, Kumamoto 861-1192, Japan
}

\begin{abstract}
Okuda, M., Matsumoto, M., Tanaka, Y., Subandiyah, S., and Iwanami, T. 2005. Characterization of the tufB-secE-nusG-rplKAJL-rpoB gene cluster of the citrus greening organism and detection by loop-mediated isothermal amplification. Plant Dis. 89:705-711.

Thermal asymmetric interlaced polymerase chain reaction (TAIL-PCR) was performed to amplify the uncharacterized regions adjacent to the nusG-rplKAJL-rpoB gene cluster of citrus greening organism (GO) isolates from different locations in Japan and Indonesia. Conventional PCR was used to amplify the internal nusG-rplKAJL-rpoB gene cluster of these isolates, and the complete sequence of this 6.1-kb fragment was determined. Comparisons with other bacterial sequences showed that the fragment is the tufB-secE-nusG-rplKAJL-rpoB gene cluster. The organization of this gene cluster is similar to that of the homologous cluster found in Escherichia coli. Except for three nucleotide changes, the sequence was identical among Japanese and Indonesian isolates. A loop-mediated isothermal amplification (LAMP) assay based on the conserved sequence of the nusG-rplKAJL-rpoB gene cluster was developed for the detection of the GO. The LAMP product was rapidly detected on nylon membranes by staining with AzurB. LAMP could detect as low as about 300 copies of the nusG-rplKAJL-rpoB fragment of the Japanese and Indonesian isolates of GO. The LAMP-based detection method, which does not depend upon a thermal cycler and electrophoresis apparatus, will be useful for under-equipped laboratories, including those found in extension centers and quarantine offices.
\end{abstract}

Additional keywords: Diaphorina citri, quantitative PCR

Citrus greening (CG, Huanglongbing) is a serious disease of citrus that limits production in many parts of Asia and Africa (2). The causal agents (citrus greening organism, GO) are designated "Candidatus Liberobacter asiaticum" and "Candidatus Liberobacter africanum" for the Asian and African greening species, respectively (6). They are nonculturable phloem-limited bacteria and are transmitted by two distinct insect vectors, Diaphorina citri and Trioza erytreae (2). The Asian greening vector, $D$. citri, is common in subtropical islands in Japan, rendering trees in those regions susceptible once the pathogen is introduced (9). The disease was found for the

Corresponding author: T. Iwanami

E-mail: tiwsw37@affrc.go.jp

The nucleotide sequence data reported in this paper will appear in the DDBJ, EMBL, and GenBank nucleotide sequence databases with the following accession number: AY342001.

Accepted for publication 15 January 2005.

DOI: 10.1094/PD-89-0705

(C) 2005 The American Phytopathological Society first time in the southernmost islands of Japan in 1988 (8). Since then, the disease has spread northward to the main production areas, posing a great threat to domestic citrus cultivation.

Studies of the GO have been hampered by the fact that it cannot be cultured on artificial media. Only a few fragments of genomic DNA have been cloned and sequenced $(4,6,12,14,15)$. The best characterized regions are $16 \mathrm{~S}$ rDNA and $16 \mathrm{~S} / 23 \mathrm{~S}$ intergenic regions $(4,6,14)$. The nucleotide sequences of these regions of several Japanese, Philippine, and Indonesian isolates are identical, similar to those from India and China, and distinct from that from Africa (14). This is consistent with the current status of the GO.

In Japan, where the disease is still limited to relatively few orchards and backyard gardens, it is managed by removal of infected trees at early stages of infection. To facilitate this practice, a rapid and reliable assay is indispensable, since the nonspecific nature of the foliar symptoms makes greening difficult to distinguish from nutrient deficiencies or other diseases. Rapid, simple, and reliable detection methods are also important in GO- devastated countries like Indonesia. Serological methods have been developed but are inconsistent due to low concentration and uneven distribution of the pathogen in infected citrus. Although amplification of the $16 \mathrm{~S}$ rDNA fragment by polymerase chain reaction (PCR) is sensitive and reproducible (5), it is applicable only in laboratories equipped with a thermal cycler and other basic apparatus for molecular biological experiments and staffed with well-trained personnel.

Loop-mediated isothermal amplification (LAMP) is a new DNA amplification method (11). This method is based on auto-cycling strand displacement DNA synthesis by a DNA polymerase, which has high strand displacement activity, and a set of specially designed inner and outer primers (11). Typically, amplification is completed within $30 \mathrm{~min}$ using a simple, affordable water bath, which is kept constantly at $65^{\circ} \mathrm{C}$. By applying LAMP, the GO might be readily detected in underequipped laboratories of extension centers and local quarantine offices.

Preliminary investigation revealed that the $16 \mathrm{~S}$ rDNA and $16 \mathrm{~S} / 23 \mathrm{~S}$ intergenic regions of GO were not suitable for LAMP, because they share homology with plant genomic DNA. In contrast, the nusG$r p l \mathrm{KAJL}-r p o \mathrm{~B}$ gene cluster (15) was more promising because of its specificity, and was chosen for this study. The objectives of this study were (i) to further characterize the nusG-rplKAJL-rpoB gene cluster by identifying the sequences adjacent to the gene cluster in several isolates from Japan and Indonesia, utilizing thermal asymmetric interlaced PCR (TAIL-PCR) (7), and (ii) to establish a LAMP-based detection method for GO using primers. This method was designed based on the sequence of the conserved region of the nusG-rplKAJL-rpoB gene cluster, and uses a simple stain to visualize products on nylon membranes.

\section{MATERIALS AND METHODS}

Sample preparation. Leaf samples were collected from infected citrus trees in different groves on subtropical and tropical islands in Japan and Indonesia (Table 1). 
All field and greenhouse samples from Japan and Indonesia were collected during summer. Total DNA was extracted from about $0.2 \mathrm{~g}$ of the leaf midrib of infected citrus using cetyltrimethylammonium bromide (CTAB), as reported previously (10), and suspended with 20 to $200 \mu \mathrm{l}$ of TE buffer (10 mM Tris-HCl, $1 \mathrm{mM}$ EDTA, $\mathrm{pH}$

Table 1. Isolates and field sources of citrus greening organism used in this study

\begin{tabular}{|c|c|c|}
\hline $\begin{array}{l}\text { Isolates and } \\
\text { field sources }^{\mathrm{a}}\end{array}$ & Location and year of collection & Host \\
\hline \multicolumn{3}{|c|}{ Japanese isolates and field sources } \\
\hline KIN1 & Kin, Okinawa, (ref. 14), 1994 & Rough lemon (Citrus jambhiri) \\
\hline OK901 & Iriomote, Okinawa (ref. 8) 1988 & Rough lemon (C. jambhiri) \\
\hline OKS13 & Ginowan, Okinawa (ref. 14), 1994 & Rough lemon (C. jambhiri) \\
\hline CG4 & Motobu, Okinawa ,2001 & Tankan (C. tankan) \\
\hline Y02-57 & Yoron, Kagoshima, 2002 & Tankan (C. tankan) \\
\hline Y02-83 & Yoron, Kagoshima, 2002 & Oto (C. oto hort. ex Y.) \\
\hline Y03-27 & Yoron, Kagoshima, 2003 & Kunenbo (C. nobilis var. kunip) \\
\hline OK03-01 & Kunigasira, Okinawa, 2003 & Shikuwasha (C. depressa) \\
\hline OK03-02 & Kunigasira, Okinawa, 2003 & Shikuwasha (C. depressa) \\
\hline \multicolumn{3}{|c|}{ Indonesian field sources } \\
\hline IDN03-2 & Seberkat, Tebes, Sambas, West Kalimantan, 2003 & Siem Pontianak (C. reticulata) \\
\hline IDN03-5 & Sindhuarjo, Ngaglik, Sleman, Yogyakarta, 2003 & Siem Mandarin (C. reticulata) \\
\hline IDN03-7 & Karangduwur, Kemili, Purworejo, Central Java, 2003 & Siem Mandarin (C. reticulata) \\
\hline
\end{tabular}

Table 2. Nucleotide sequences and positions of primers used for TAIL-PCR and LAMPa

\begin{tabular}{|c|c|c|}
\hline Name & Sequence & Position $^{b}$ \\
\hline \multicolumn{3}{|c|}{ TAIL-PCR primers } \\
\hline Tail2 & 5'-GTNCGASWCANAWGTT-3' & \\
\hline GSFW6 & 5'-CGTCTCGTCAAGATTGCTATCCG-3' & $3672-3694$ \\
\hline GSFW7 & 5'-GCAAGGTTAGTTCTGTTCATCG-3' & $4016-4037$ \\
\hline GSFW9 & 5'-TCCCGATGAGCGTCCTAATG-3' & $4704-4723$ \\
\hline GSRV3 & 5'-CGAAGAAAACAGACGATACAGGC-3' & $1951-1929$ \\
\hline GSRV4 & 5'-GACCATCAGAGACACAAACC-3' & $1984-1965$ \\
\hline GSRV5 & 5'-TAACTCTACTGGTGTGACACGAC-3' & $2085-2063$ \\
\hline \multicolumn{3}{|c|}{ PCR primers } \\
\hline PCFW13 & 5'-GCCTGTATCGTCTGTTTTCTTCG-3' & $1929-1951$ \\
\hline PCFW1 & 5'-GTCATCGCATATGGGATGTGGT-3' & $3280-3301$ \\
\hline PCRV2 & 5'-ATTTTAGGAGCAATAACAGGAC-3' & $3781-3760$ \\
\hline PCRV1 & 5'-CGTCTTTAGTCATAAGAG-3' & $4990-4973$ \\
\hline \multicolumn{3}{|c|}{ Sequence primers } \\
\hline SQFW14 & 5'-GTTAAGTTGCAGATAGAGTCGGG-3' & $2230-2252$ \\
\hline SQFW15 & 5'-GAAGGTTCGGCCTGTTCAATGG-3' & $2593-2614$ \\
\hline SQFW16 & 5'-GAAATTGTTAAAGGCGGGCAG-3' & $2942-2962$ \\
\hline SQFW8 & 5'-GAAGATGCTGGTGCAACGTAG-3' & $4422-4443$ \\
\hline SQFW10 & 5'-ATGGGCGATCTTCCTCTTATGAC-3' & $4960-4982$ \\
\hline SQFW11 & 5'-TCСТАAАATCGTCTATTCACAAAG-3' & $5265-5288$ \\
\hline SQFW12 & 5'-GTTGTCCGCTGCCGATTTGATG-3' & $5310-5331$ \\
\hline SQFW19 & 5'-GTCGATTCTGTGAATAATAATGC-3' & $5611-5633$ \\
\hline SQRV9 & 5'-TCTCCACATCTGTACACTTGAC-3' & $718-697$ \\
\hline SQRV10 & 5'-AACATCCGCACGATTTACTCC-3' & $798-778$ \\
\hline SQRV7 & 5'-AACTCTCCAATCTTAGAACACGC-3' & $1347-1325$ \\
\hline SQRV8 & 5'-TAGACTCATCCCTAACTTGC-3' & 1414-1395 \\
\hline \multicolumn{3}{|c|}{ LAMP primers for $r p l \mathrm{KAJL}-r p o \mathrm{~B}$ operon } \\
\hline rpl-FIP & $\begin{array}{l}\text { 5'-GCATGCCGAGGATCAATGCCT-TGCTTAAAGAGCGTGC- } \\
\text { TACG-3' }\end{array}$ & $2727-2802$ \\
\hline rpl-BIP & $\begin{array}{l}\text { 5'-TATGCCTAATGGCACGGGGGTA-AGCTTCATCCGCCTT- } \\
\text { CGA-3' }\end{array}$ & $2899-2830$ \\
\hline rpl-F3 & 5'-TGGGTTAAGTGATGCTGTGG-3' & $2701-2721$ \\
\hline rpl-B3 & 5'-CAACAATATCAGCCCCTGCT-3' & 2924-2905 \\
\hline \multicolumn{3}{|c|}{ LAMP primers for $16 \mathrm{SrDNA}$ region } \\
\hline rdna-FIP & $\begin{array}{l}\text { 5'-TCCGCGATTACTAGCGATTCCGCTCAGTTCGGATTGCA- } \\
\text { CTCT-3' }\end{array}$ & $45-106$ \\
\hline rdna-BIP & 5'-TCAGCATGCCGCGGTGAATAAGGCAAAACCAACTCCCA-3' & $180-107$ \\
\hline rdna-F3 & 5'-AGCTAATCCCCAAAAGCCAT-3' & $25-46$ \\
\hline rdna-B3 & 5'-CCTCCTTGCGGTTAGCAC-3' & $85-106$ \\
\hline \multicolumn{3}{|c|}{$\begin{array}{l}\text { a TAIL-PCR = thermal asymmetric interlaced polymerase chain reaction; LAMP = loop-mediated } \\
\text { isothermal amplification. } \\
\text { b Nucleotide positions are numbered following the sequence in the GenBank database AY342001 for } \\
\text { TAIL-PCR primers and LAMP primers for nusG-rplKAJL-rpoB gene cluster, and AB } 019793 \text { for } \\
\text { LAMP primers for 16SrDNA region. Nucleotide positions of FIP and BIP indicate target regions of } \\
\text { the gene cluster, which do not match perfectly with the sequences of the primers because of the pecu- } \\
\text { liar structure of the primers. }\end{array}$} \\
\hline
\end{tabular}

8.0). DNA of the established isolates KIN1, OK901, and OKS13 was purified from the infected rough lemon (Citrus jambhiri), which were grown in a greenhouse at $30^{\circ} \mathrm{C}$ day and $25^{\circ} \mathrm{C}$ night.

TAIL-PCR and sequencing of the region adjacent to the tufB-secE-nusGrplKAJL-rpoB gene cluster. TAIL-PCR (7) was performed to determine the nucleotide sequences of the regions adjacent to the nusG-rplKAJL-rpoB gene cluster. The isolate KIN1 was used for the TAILPCR experiments. Specific primers GSRV3, GSRV4, and GSRV5 for the region upstream of the known sequence, and GSFW6, GSFW7, and GSFW9 for the region downstream, were used in combination with a short arbitrary primer TAIL2 (Table 2, Fig. 1). The reaction mixtures and thermal cycling conditions are as described previously (7). Conventional PCR was used to amplify the internal nusG$r p l \mathrm{KAJL}-r p o \mathrm{~B}$ gene cluster, using a primer set of PCFW13 and PCRV2 or PCFW1 and PCRV1, which were designed from the nucleotide sequence of the Indian isolate (GenBank accession no. M94319) and shown in Table 2. PCR reaction mixture consisted of $1 \times$ PCR buffer $(10 \mathrm{mM}$ TrisHCL, $50 \mathrm{mM} \mathrm{KCl}, 2.5 \mathrm{mM} \mathrm{MgCl} 2, \mathrm{pH}$ 8.3), $200 \mu \mathrm{M}$ of each dNTP, 2.5 units of AmpliTaq DNA polymerase (Applied Biosystems, Foster City, CA), $1 \mu \mathrm{M}$ each of forward and reverse primers, and an arbitrary amount of DNA template, which had been prepared from the CG-infected citrus leaves from Japan and Indonesia. The amplifications were performed in a DNA Thermal Cycler 9600 (Applied Biosystems) for 40 cycles under the following conditions: $30 \mathrm{~s}$ at $94^{\circ} \mathrm{C}, 30 \mathrm{~s}$ at 50 to $60^{\circ} \mathrm{C}$, depending on the $\mathrm{Tm}$ of the primers, and $60 \mathrm{~s}$ at $72^{\circ} \mathrm{C}$ with an initial denaturation step of $2 \mathrm{~min}$ at $94^{\circ} \mathrm{C}$. Both TAILPCR and PCR products were directly sequenced by dideoxy-mediated chain termination (Sanger) method (13) using one of the PCR or sequence primers shown in Table 2, a DNA thermal sequencing kit (Applied Biosystems), and a DNA sequencer model 373S (Applied Biosystems). For the other isolates from Japan and Indonesia, PCR was used to amplify the tufB-secE-nusG-rplKAJL-rpoB gene cluster using primers that were designed from the newly determined sequence of KIN1. These PCR products were directly sequenced.

LAMP. LAMP reactions were conducted as reported previously (11), in $10 \mu \mathrm{l}$ of the mixture containing $1 \times$ reaction buffer $(20 \mathrm{mM}$ Tris- $\mathrm{HCl}, 10 \mathrm{mM} \mathrm{KCl}, 8$ $\mathrm{mM} \mathrm{MgSO} 4,10 \mathrm{mM}\left(\mathrm{NH}_{4}\right)_{2} \mathrm{SO}_{4}, 0.1 \%$ Tween 20, 0.8 M betaine, and $1.4 \mathrm{mM}$ each of dNTPs) together with $8 \mathrm{U}$ of $B s t$ DNA polymerase (New England Biolabs, Beverly, MA), $1.6 \mu \mathrm{M}$ each of FIP and BIP primers, $0.2 \mu \mathrm{M}$ each of $\mathrm{F} 3$ and $\mathrm{B} 3$ primers, and $1 \mu \mathrm{l}$ of the total DNA from infected citrus. FIP, BIP, F3, and B3 for am- 
plification of a part of the rplKAJL-rpoB operon or the $16 \mathrm{~S}$ rDNA region were designed using a software PrimerExplore V1 (Fujitsu, Tokyo, Japan; Table 2). Template DNA and primers were denatured at $95^{\circ} \mathrm{C}$ for $5 \mathrm{~min}$ before the other reagents were added. The reaction mixture was incubated at $65^{\circ} \mathrm{C}$ for up to $1 \mathrm{~h}$.

Comparison of sensitivity of LAMP and PCR. Total DNA from infected citrus leaves was prepared in 10-fold dilution series of $100 \mathrm{ng}, 10 \mathrm{ng}, 1 \mathrm{ng}, 100 \mathrm{pg}(0.1$ $\mathrm{ng})$, and $10 \mathrm{pg}(0.01 \mathrm{ng})$ per microliter with TE. Using these templates, LAMP was carried out as described above. PCR with GO-specific primers rpl-F3 and rplB3 (Table 2) was performed with the thermal cycling condition of 35 cycles of $94^{\circ} \mathrm{C}$ for $30 \mathrm{~s}, 58^{\circ} \mathrm{C}$ for $30 \mathrm{~s}$, and $72^{\circ} \mathrm{C}$ for $30 \mathrm{~s}$ after initial denaturation for $2 \mathrm{~min}$. The sensitivity of LAMP was compared with that of PCR.

Visualization of the amplification product by staining. One microliter of DNA dilution series $(1,10$, and $100 \mathrm{ng} / \mu \mathrm{l})$ of citrus genomic DNA (template) or LAMP products were spotted onto nylon membranes (Hybond N+, Amersham Bioscience, Buckinghamshire, UK). The DNA was denatured and fixed to the membrane by soaking in $0.4 \mathrm{M} \mathrm{NaOH}$ for $5 \mathrm{~min}$, followed by a brief wash in $2 \times \operatorname{SSC}(0.3 \mathrm{M}$ $\mathrm{NaCl}$ and $0.03 \mathrm{M}$ sodium citrate). The membranes were air-dried on paper towels and then soaked in $0.1 \%$ AzurB, $0.1 \%$ methylene blue, $0.1 \%$ crystal violet, or $1 \times$ Mupid Blue (ADVANCE-BIO, Tokyo, Japan) until spots became visible, typically 1 to $2 \mathrm{~min}$. The membranes were rinsed with $50 \%$ ethanol and dried.

Sensitivity of LAMP for the GO. The concentration of the GO in total DNA extract was estimated by quantitative PCR using SYBR Green I and Light Cycler system (Roche, Indianapolis, IN). A portion of the nusG-rplKAJL-rpoB region was amplified by PCR with GO-specific primers fp1898 and rp1897 (15), and the product was purified using the Montage PCR purification kit (Millipore, Billerica, MA). Tenfold dilution series (1 ng to 0.1 fg per microliter) of the purified product were prepared by diluting with TE after estimating the original concentration with a spectrophotometer. Quantitative PCR was performed on the dilution series, and the standard curve, which relates the crossing cycle number to the logarithms of concentration of the template, was generated. Subsequently, 1 or $10 \mathrm{ng}$ of total DNA from healthy rough lemon was mixed with a diluted series of the template fragment to examine whether DNA of the healthy rough lemon leaves affects the quantitative analysis. After confirming that host DNA has little effect on amplification (see results), quantitative PCR was carried out using the total DNA sample from the GOinfected tissue, and the concentration of GO in the sample was estimated from the standard curve. The conditions of the quantitative PCR were as follows: $1 \times$ reaction mixtures (LightCycler-DNA master mix SYBR Green I, Roche) containing 100 ng of sample DNA and specific primers rpl-F1 (5'-TTTCGTTGGGCAGTCT-3') and rpl-R1 (5'-CCAGCTCGAATACCCT$\left.3^{\prime}\right)$ were put into glass capillaries, and thermal cycling reactions were performed. The primers of rpl-F1 and rpl-R1 were designed from the nucleotide sequence of the nusG-rplKAJL-rpoBC gene cluster (GenBank accession no. M94319; nucleotide positions 424 to 439 and 594 to 609 , respectively). Reaction conditions were 40 cycles of $95^{\circ} \mathrm{C}$ for $15 \mathrm{~s}, 60^{\circ} \mathrm{C}$ for $5 \mathrm{~s}$, and $72^{\circ} \mathrm{C}$ for $20 \mathrm{~s}$. The fluorimetric intensity of SYBR Green I was measured at $497 \mathrm{~nm}$ during each cycle at $72^{\circ} \mathrm{C}$.

\section{RESULTS}

TAIL-PCR and sequence of the tufBsecE-nusG-rplKAJL-rpoB gene cluster. No specific bands were observed after agarose electrophoresis of the first and second TAIL-PCR (Fig. 1B, lanes R5, R4, F6, and F7), presumably due to the low concentration of the GO DNA. A GOspecific band of about $1.9 \mathrm{kbp}$ was observed in agarose gels after the third TAILPCR with specific primer GSRV3 and the arbitrary primer TAIL2, preceded by the first and second TAIL-PCR using the primer sets GSRV5-TAIL2 and GSRV4TAIL2, respectively (Fig. 1B). There was
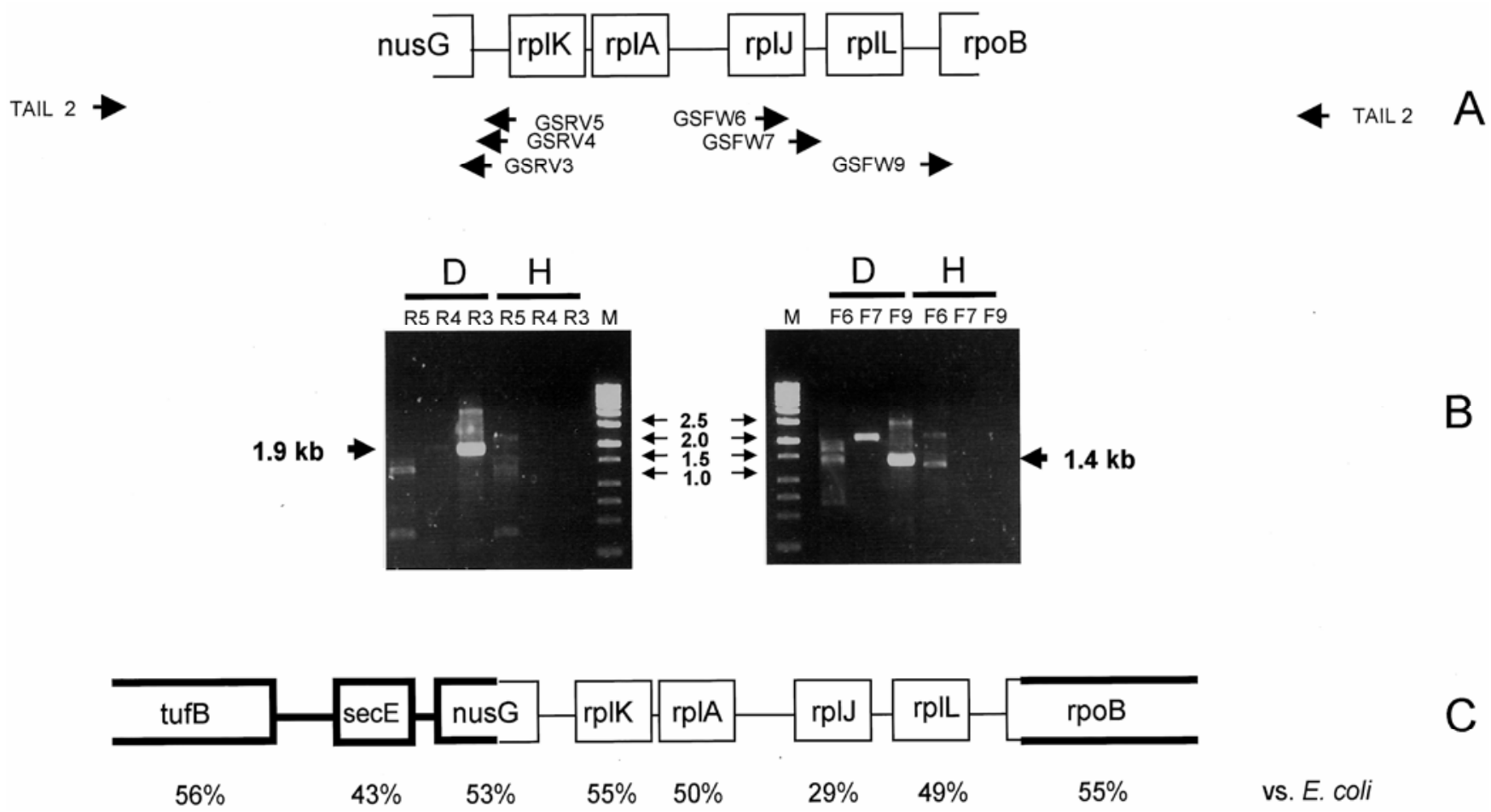

$56 \%$

$43 \%$

vs. E. coli

Fig. 1. Amplification of the region adjacent to the nusG-rplKAJL-rpoB gene cluster of the citrus greening organism (GO). A, Annealing positions of the primers used for the specific amplification of the upstream region (TAIL2, GSRV5, GSRV4, GSRV3) and the downstream region (TAIL2, GSFW6, GSFW7, GSFW9). B, Agarose gel electrophoresis of TAIL-PCR products with primer TAIL2 and one of the following specific primers: GSRV5 (indicated as R5), GSRV4 (R4), GSRV3 (R3), GSFW6 (F6), GSFW7 (F7), and GSFW9 (F9). D and H indicate infected tissue and healthy controls, respectively. C, Gene organization of the GO in the vicinity of the nusG-rplKAJL-rpoB gene cluster region revealed by thermal asymmetric interlaced polymerase chain reaction (TAIL-PCR). The regions adjacent to the nusG-rplKAJL-rpoB gene cluster elucidated in this study are shown by thick lines. Sequence identities (\%) of the putative genes with the corresponding genes of Escherichia coli are shown. 
an expected overlap of nucleotide sequence between the TAIL-PCR fragment and the $5^{\prime}$ region of the nusG-rplKAJLrpoB gene cluster, showing the authentic-

ity of the TAIL-PCR product. Likewise, a fragment of about $1.4 \mathrm{kbp}$, which is located in the $3^{\prime}$ region of the nusG$r p l \mathrm{KAJL}-r p o \mathrm{~B}$ gene cluster, was specifi-

Table 3. Differences in the tufB-secE-nusG-rplKAJL-rpoB gene cluster nucleotide sequence among the Japanese (KIN1), Indonesian (IDN03-2), and Indian (Poona) isolates of the citrus greening organism

\begin{tabular}{|c|c|c|c|c|c|c|}
\hline \multirow[b]{2}{*}{ Isolate } & \multicolumn{6}{|c|}{ Nucleotide positions ${ }^{\mathrm{a}}$ and gene } \\
\hline & $1806(n u s G)$ & $4439(r p l \mathrm{~L})$ & $4452(r p l \mathrm{~L})$ & 4453(rplL) & 5012(rpoB) & 6107(rpoB) \\
\hline KIN1 & G & $\mathrm{C}$ & $\mathrm{T}$ & A & $\mathrm{C}$ & A \\
\hline IDN03-2 & $\mathrm{T}$ & $\mathrm{C}$ & $\mathrm{T}$ & A & $\mathrm{T}$ & $\mathrm{G}$ \\
\hline Poona & n.a. ${ }^{b}$ & $-^{\mathrm{c}}$ & A & $\mathrm{T}$ & $\mathrm{C}$ & n.a. \\
\hline
\end{tabular}

a Nucleotide positions are numbered following the sequence in the GenBank database AY342001.

${ }^{\mathrm{b}}$ n.a. = sequence not available for comparison.

$c_{-}=$gap in the sequence.

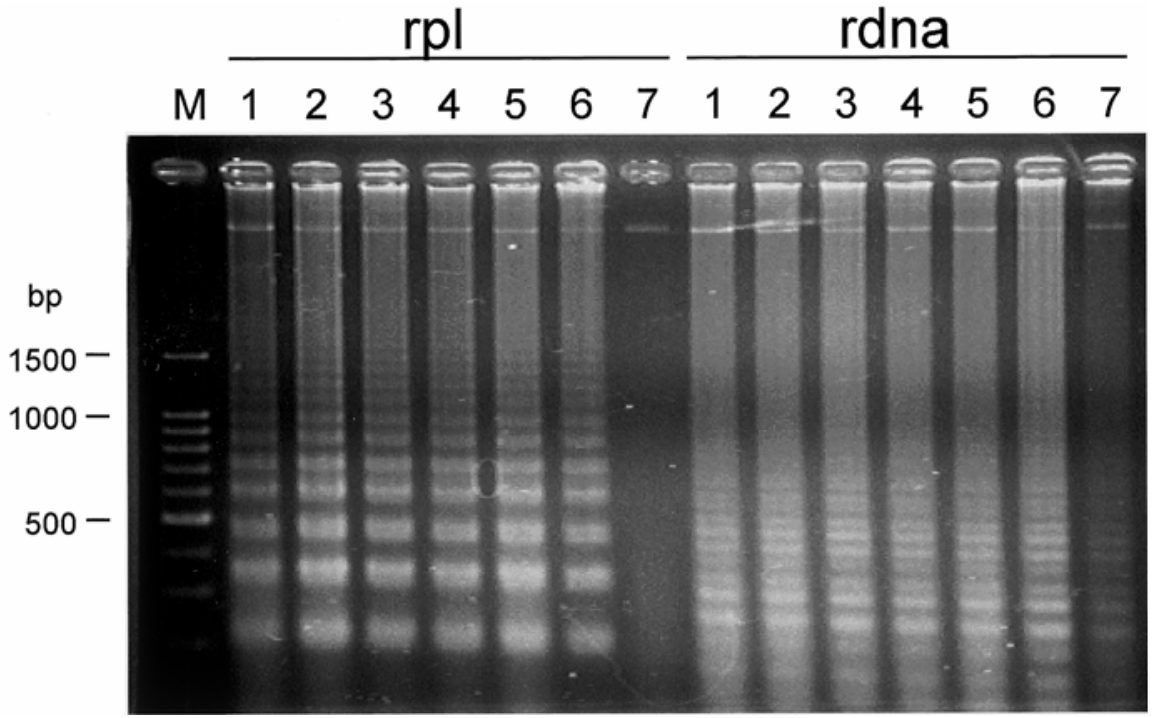

Fig. 2. Agarose gel electrophoresis of loop-mediated isothermal amplification (LAMP) products amplified with the rpl primer set (rpl) or 16SrDNA primer set (rdna). $100 \mathrm{ng}$ DNA of total DNA from citrus infected with KIN1 (lane 1), OKS13 (lane 2), Y02-57 (lane 3), IDN03-2 (lane 4), IDN03-5 (lane 5), IDN03-7 (lane 6), and from healthy rough lemon (lane 7) were used as templates, respectively. M indicates DNA size markers.

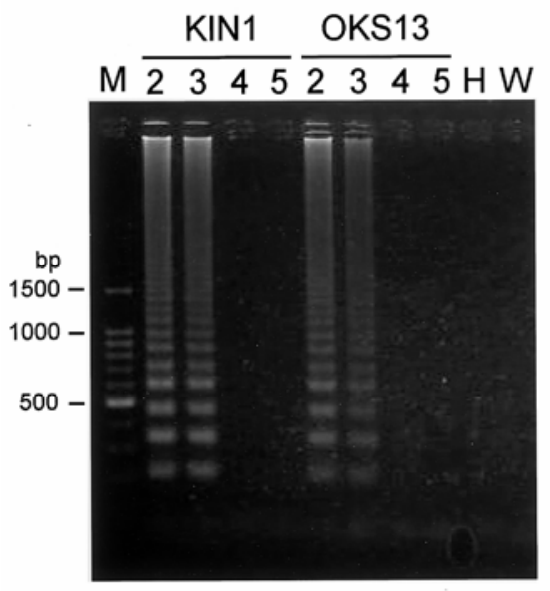

A

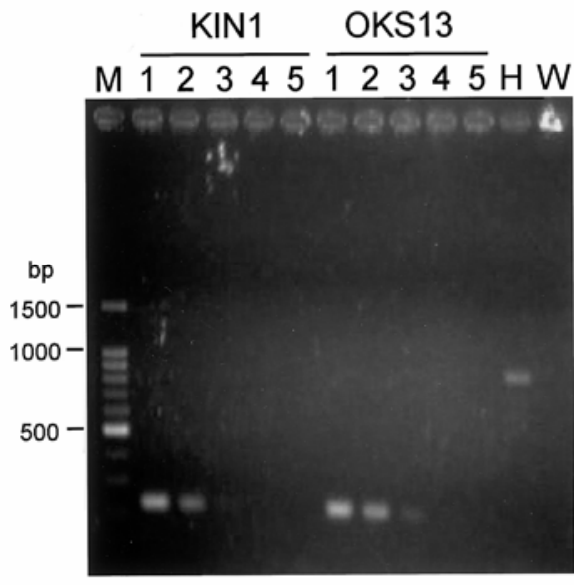

B
Fig. 3. Agarose gel electrophoresis of loop-mediated isothermal amplification (LAMP) products amplified with the rpl primer set for the $r p l \mathrm{KAJL}-r p o \mathrm{~B}$ operon (A) and polymerase chain reaction (PCR) products amplified with primers F3 and B3 (B). Total DNA of citrus greening organism (GO)-infected leaves (KIN1 and OKS13) were diluted and used as templates: 1, $100 \mathrm{ng} ; 2$, $10 \mathrm{ng} ; 3$, $1 \mathrm{ng}$; 4, $0.1 \mathrm{ng}$; $5,0.01 \mathrm{ng}$ per tube. $\mathrm{H}$ and $\mathrm{W}$ are $10 \mathrm{ng}$ of total DNA of healthy rough lemon and sterile water, respectively. $\mathrm{M}$ indicates DNA size markers. cally amplified by TAIL-PCR using the primers GSFW6, GSFW7, and GSFW9 in combination with the arbitrary primer TAIL2 (Fig. 1). In addition to these TAILPCR amplifications, the internal known region of nus G-rplKAJL-rpoB gene cluster was amplified by PCR, and the overall sequence was determined completely, resulting in a fragment of $6,145 \mathrm{bp}$ with a $\mathrm{G}+\mathrm{C}$ content of $39.7 \%$. Comparison with the nucleotide sequences in the DNA data bank of Japan (DDBJ) suggested that the DNA fragment contains the tufB-secEnus G-rplKAJL-rpoB gene cluster, which is reminiscent of that of Escherichia coli (3). The percentages of sequence identities between GO and E. coli of each gene are shown in Figure 1.

The 6.1-kbp fragment of the tufB-sec $\mathrm{E}-$ $n u s \mathrm{G}-r p l \mathrm{KAJL}-r p o \mathrm{~B}$ was amplified by PCR from another two Japanese isolates (OK901 and Y02-57) as well as three Indonesian isolates (In03-2, In03-5, and In03-7; Table 1). The sequences of the isolates within each country were identical, while there were three nucleotide changes at nucleotide positions 1806, 5012, and 6107 between Japanese GO and Indonesian GO (Table 3).

The sequences of a partial fragment (3.0 $\mathrm{kb}$ ) of the nusG-rplKAJL-rpoB gene cluster of Indian GO as well as a shorter fragment $(1.7 \mathrm{~kb})$ of the rplKAJL-rpoB operon of African GO had been reported previously $(12,15)$. When compared with Indian GO, there are three nucleotide changes, at nucleotide positions 4439,4452 , and 4453 , between Japanese and Indian GO, while the sequence was conserved between Japanese and Indonesian GO in these positions (Table 3). Interestingly, all these nucleotide changes were found in the putative genes, not in the intergenic spacer region (Table 3 ). The sequence of the rplKAJL-rpoBC operon of both Japanese and Indonesian GO showed $81 \%$ identity overall at the nucleotide level with that of African GO.

LAMP and its sensitivity. When suitable primer sets were searched, the computer program PrimerExplore V1 showed several candidates for both the rplKAJLrpo $\mathrm{BC}$ operon and the 16SrDNA region. A combination of primers, which had the least probability of dimer formation, was selected for each region, and further experiments were conducted using these primer sets (Table 2).

Typical ladder-like bands were observed after agarose gel electrophoresis of the LAMP products of the $r p l \mathrm{KAJL}-r p o \mathrm{BC}$ operon, suggesting successful amplification of the targeted region (Fig. 2). Sufficient amounts of products for agarose gel electrophoresis were obtained in $30 \mathrm{~min}$ of incubation. The specific amplification was observed from all field isolates, which had tested positive in PCR detection, as well as from the established isolates kept in the greenhouse (Fig. 2). Ladder-like bands were not observed in agarose gel electro- 
phoresis, when healthy rough lemon DNA was used as template (Fig. 2). When LAMP was tried for a 16S rDNA fragment, ladder-like bands were not as clear as those of the $r p l \mathrm{KAJL}-r p o \mathrm{BC}$ operon, and some nonspecific bands were also present in reactions of the healthy rough lemon DNA (Fig. 2).

LAMP and PCR were tested for 10, 1 , 0.1 , and $0.01 \mathrm{ng}$ of the total DNA from citrus, which was infected with KIN1 or OKS13 isolate. Both LAMP and PCR could detect GO-specific DNA fragments from 10 and $1 \mathrm{ng}$ of the total DNA (Fig. 3), suggesting that LAMP is at least as sensitive as PCR.

Visualization by staining with dyes. In the preliminary experiment, amounts greater than $10 \mathrm{ng}$ of genomic or LAMPamplified DNA were clearly visualized by spotting nylon membranes and staining with AzurB or Mupid Blue, but not by crystal violet or methylene blue, in $2 \mathrm{~min}$ (data not shown). The results indicated that GO DNA can be visualized on the membrane by AzurB and Mupid Blue without background staining of the host DNA when the GO DNA is amplified to the concentration of over $10 \mathrm{ng} / \mu \mathrm{l}$ from reaction mixture that contains less than 10 $\mathrm{ng} / \mu \mathrm{l}$ of the total citrus DNA.

AzurB was used in further experiments, because it is more commonly available than Mupid Blue. After staining, the GOspecific LAMP products, which were amplified from 1 or $10 \mathrm{ng}$ of total DNA, were observed as distinct spots on the membrane. Aliquots from LAMP reactions that contained DNA from healthy rough lemon and sterile water were not stained at all, and samples that contained less than $1 \mathrm{ng}$ DNA of GO-infected citrus leaves were stained only faintly (Fig. 4). Ladder-like DNA bands were not observed after agarose gel electrophoresis of the LAMP products from these samples (Fig. 3). When less than $10 \mathrm{ng}$ of total DNA extracted from the GO-infected citrus in the fields of Japan and Indonesia were constantly used in LAMP reaction, the products from these samples were stained specifically on the nylon membrane (Figs. 4 and 5). The initial denaturation step at $95^{\circ} \mathrm{C}$ for $5 \mathrm{~min}$ was indispensable for stable LAMP amplification from field samples (data not shown).

Quantification of sensitivity of LAMP. As shown in Table 4, the presence of healthy DNA had little effect on the crossing points in quantitative PCR analysis. When quantitative PCR was conducted using $100 \mathrm{ng}$ of total DNA extracted from rough lemon infected with KIN1 and OKS13, the numbers of cycles at the crossing point were 21.32 and 21.28 , respectively. The standard curve generated from a diluted series of the partial nusG-rplKAJL$r p o \mathrm{~B}$ region was valid $(r=-0.98)$ (Fig. 6), and the amounts of GO molecules were calculated to be $2.81 \times 10^{4}$ and $2.88 \times 10^{4}$ copies per $100 \mathrm{ng}$ of total DNA for KIN1 and 0KS13 isolates, respectively, based on the assumption that the molecular weight of the $r p l$ fragment was $1,056,333$. Since LAMP could detect GO from these samples when $1 \mathrm{ng}$ of the total DNA was used as templates, as shown in the sensitivity test (Figs. 3 and 4), it was concluded that LAMP could detect 281 to 288 (ca. 300) copies of the nusG-rplKAJL-rpoB fragment of GO.

\section{DISCUSSION}

This study showed that there are two putative genes of tuf $\mathrm{B}$ and $\sec \mathrm{E}(1)$ in the upstream of the $n u s \mathrm{G}-r p l \mathrm{KAJL}-r p o \mathrm{~B}$ gene cluster of GO. The results suggested that the organization of this region of the GO

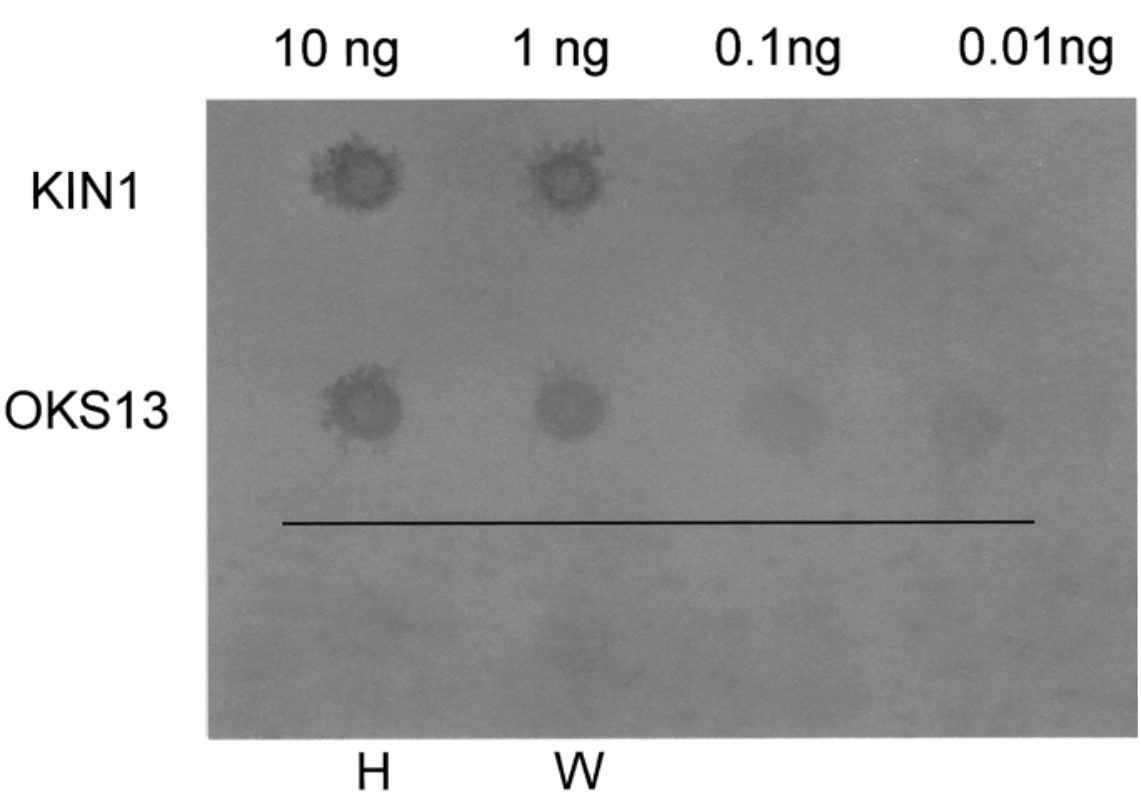

Fig. 4. Colorimetric visualization of loop-mediated isothermal amplification (LAMP) products amplified with the rpl primer set. One microliter of amplified DNA was fixed on nylon membrane and stained with $0.1 \%$ AzurB. $\mathrm{H}$ and $\mathrm{W}$ indicate aliquots from LAMP reactions from healthy rough lemon DNA and sterile water, respectively. 10, 1, 0.1, or $0.01 \mathrm{ng}$ of total DNA from KIN1- or OKS13infected tissue was used for amplification by LAMP.
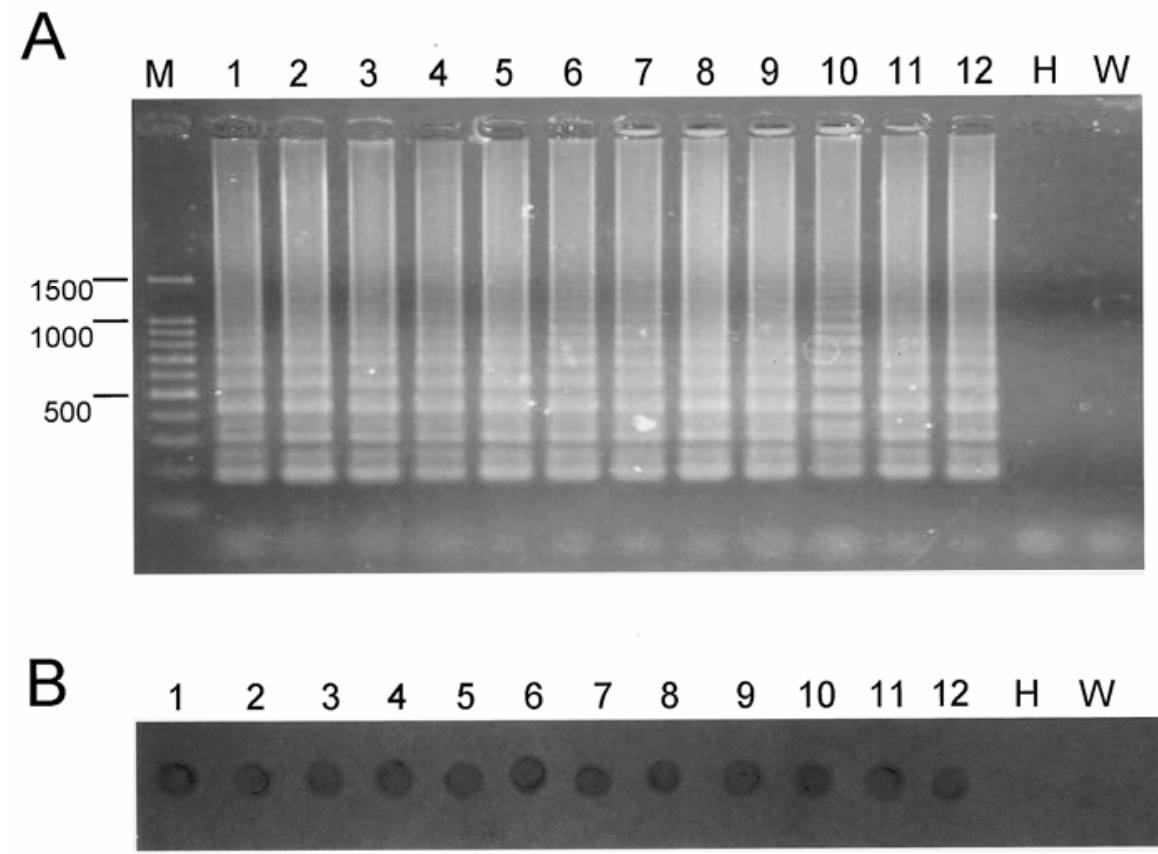

Fig. 5. Detection of the greening organism from citrus leaves grown in fields and greenhouse. Agarose gel electrophoresis (A) and staining of the nylon membrane with AzurB (B) of $1 \mu$ l of the loopmediated isothermal amplification (LAMP) product. For both $\mathbf{A}$ and $\mathbf{B}$, lanes 1 to 12 are KIN1, OK901, OKS13, CG4, Y02-57, Y02-83, Y03-27, OK03-01, OK03-01, IDN03-2, IDN03-5, and IDN03-7. $\mathrm{H}$ and $\mathrm{W}$ are $10 \mathrm{ng}$ of total DNA of healthy rough lemon and sterile water, respectively. $\mathrm{M}$ indicates DNA size markers. Isolates are described in Table 1. 
genome is similar to that of the homologous region of $E$. coli. The $\mathrm{G}+\mathrm{C}$ content of the $6.1-\mathrm{kb}$ fragment of the tufB-sec $\mathrm{E}-$ $n u s \mathrm{G}-r p l \mathrm{KAJL}-r p o \mathrm{~B}$ gene cluster is $39.7 \%$, in agreement with the previous report (15).

Previously, we found that the sequences of the $16 \mathrm{~S}$ rDNA and $16 \mathrm{~S} / 23 \mathrm{~S}$ intergenic regions were identical among Japanese and Indonesian isolates (14). The sequences of these regions were also identical among the new Japanese (Y02-57) and Indonesian IDN03-7) collected in this study (data not shown). It had been impossible to distinguish Japanese isolates from Indonesian ones on the basis of the nucleotide sequence of these regions. In contrast, there are three nucleotide differences between Japanese isolates and Indonesian isolates in the tufB-secE-nusG-rplKAJL-rpoB gene cluster, suggesting that this fragment might be utilized for differentiating strains. On the other hand, with the exceptions of field sources (IDN03-2, IDN03-5, and

these three nucleotide changes as well as a few differences found in the sequence of the region in the Indian isolate, the sequence of the tufB-secE-nusG-rplKAJL$r p o B$ gene cluster is highly conserved among Asian GO and is a suitable target for LAMP detection.

The results of this study show that LAMP-based detection of the GO using an rpl primer set for the rplKAJL-rpoB operon is highly sensitive and specific. It is very likely applicable to most of the Asian GO. All samples were collected during summer in this study. Samples collected during other seasons and from other regions of Asia are yet to be tested. LAMPbased detection for GO was sensitive enough to detect as few as 300 copies of $n u s \mathrm{G}-r p l \mathrm{KAJL}-r p o \mathrm{~B}$ gene molecules in a sample tube. We note that LAMP could detect as few as six copies of the HBs region of hepatitis virus $B$, in the presence of $100 \mathrm{ng}$ of human genomic DNA (11). The reasons for the difference in sensitivity are

Table 4. Effect of healthy citrus DNA on crossing point of quantitative analysis of rpl region of greening organism

\begin{tabular}{cccc}
\hline & \multicolumn{3}{c}{ Crossing point } \\
\cline { 2 - 4 } DNA of rpl fragment & $\mathbf{0 ~ n g / t u b \mathbf { a } ^ { \mathbf { a } }}$ & $\mathbf{1 ~ \mathbf { ~ n g } / \text { tube }}$ & $\mathbf{1 0} \mathbf{~ n g} /$ tube \\
\hline $1 \mathrm{ng}$ & 5.30 & 5.24 & 5.25 \\
$100 \mathrm{pg}$ & 7.89 & 7.96 & 8.07 \\
$10 \mathrm{pg}$ & 11.37 & 11.37 & 11.63 \\
$1 \mathrm{pg}$ & 14.85 & 14.76 & 14.72 \\
$100 \mathrm{fg}$ & 19.08 & 18.33 & 18.78 \\
$10 \mathrm{fg}$ & 21.90 & 22.16 & 22.06 \\
$1 \mathrm{fg}$ & 24.68 & 24.34 & 24.52 \\
\hline
\end{tabular}

${ }^{a}$ Amount of healthy rough lemon DNA contained in a tube.

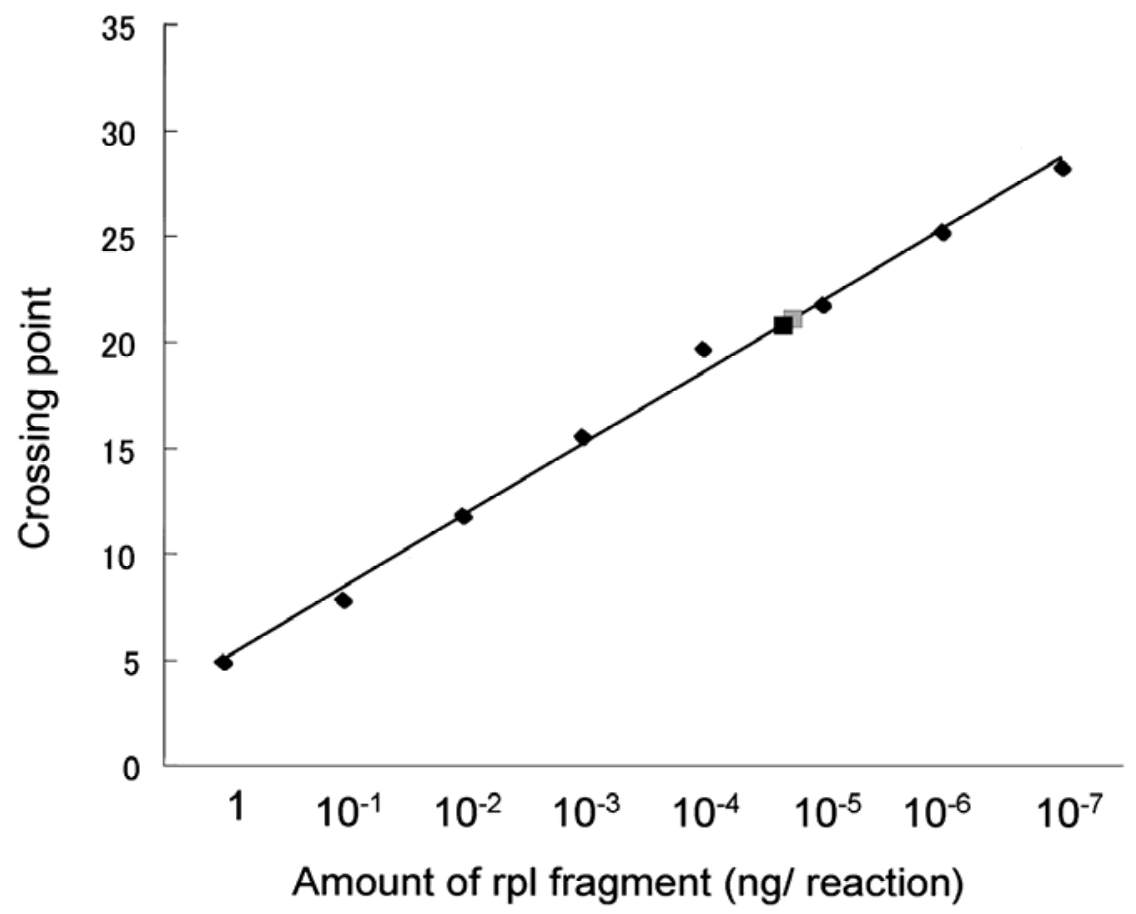

Fig. 6. Standard curve of quantitative polymerase chain reaction (PCR) from samples of known concentration of $r p l \mathrm{KAJL}-r p o \mathrm{~B}$ operon fragment. Crossing points of the total plant DNA (100 ng/ $\mu \mathrm{l})$ of KIN1 and OKS13 were indicated by black and gray squares, respectively. not clear. The difference might be due to inhibition of Bst DNA polymerase activity by some substances in the DNA samples or due to a reduced efficiency of DNA extension arising from the secondary structure of the target region. Also, designing better sets of primers might increase sensitivity of LAMP-based detection of the GO. However, LAMP-based detection of the GO developed in this study is as sensitive as the conventional PCR assay, which is sufficient for practical diagnosis. In some instances, extreme sensitivity may even be detrimental, since it leads to false positive results due to contamination of samples. LAMP-based detection methodology will be very applicable in under-equipped facilities and extension centers, especially when combined with colorimetric visualization using AzurB. The staining of a nylon membrane replaces conventional electrophoresis and visualization of DNA bands under UV illumination. In conclusion, the newly determined sequence of the $t u f \mathrm{~B}-s e c \mathrm{E}-n u s \mathrm{G}-r p l \mathrm{KAJL}-r p o \mathrm{~B}$ gene cluster of the GO revealed an E. coli-like gene organization. This region also provides a conserved and specific region, which was used for LAMP-based detection. The LAMP protocol and subsequent color development of the products on the nylon membrane are not only simple but also very rapid, and will be widely applicable.

\section{ACKNOWLEDGMENTS}

The isolates from Indonesia were collected under the ACIAR project CS $2 / 2000 / 043$. We thank Andrew Beattie, University of Western Sydney, the project leader who gave permission for the team to send samples for this molecular characterization. We also thank S. Hashimoto, A. Hamashima, K. Takaesu, and K. Nasu for providing the field GO samples in Japan. We are grateful to J. Hartung for critical reading of the manuscript. This work was supported by the "Innovation of Agriculture" program (No. 1529) from the Ministry of Agriculture, Fishery and Forestry of Japan.

\section{LITERATURE CITED}

1. An, G., and Friesen, J. D. 1980. The nucleotide sequence of tufB and four nearby tRNA structure genes of Escherichia coli. Genes 12:33-39.

2. da Graca, J. V. 1991. Citrus greening disease Annu. Rev. Phytopathol. 29:109-136.

3. Downing, W. L., Sullivan, S. L., Gottesman, M. E., and Dennis, E. 1990. Sequence and transcriptional pattern of the essential E. coli sec $\mathrm{E}-n u s \mathrm{G}$ operon. J. Bacteriol. 172:16211627.

4. Jagoueix, S., Bové, J. M., and Garnier, M. 1994. The phloem-limited bacterium of greening disease of citrus is a member of the $\alpha$ subdivision of the Protobacteria. Int. J. Syst. Bacteriol. 44:379-386.

5. Jagoueix, S., Bové, J. M., and Garnier, M. 1996. PCR detection of the two 'Candidatus' liberobacter species associated with greening disease of citrus. Mol. Cell. Probes 10:43-50.

6. Jagoueix, S., Bové, J. M., and Garnier, M 1997. Comparison of the $16 \mathrm{~S} / 23 \mathrm{~S}$ ribosomal intergenic regions of "Candidatus Liberobacter asiaticum" and "Candidatus liberobacter africanum," the two species associated with citrus Huanglongbing (greening) disease. Int. J. Syst. Bacteriol. 47:224-227.

7. Liu, Y. G., and Whitttier, R. F. 1995. Thermal asymmetric interlaced PCR: Automable ampli- 
fication and sequencing of insert end fragments from P1 and YAC clones for chromosome walking. Genomics 25:674-681.

8. Miyakawa, T., and Tsuno, K. 1989. Occurrence of citrus greening in the southern islands of Japan. Ann. Phytopathol. Soc. Jpn. 66:667670.

9. Miyatake, Y. 1965. Notes on the Psllidae from the Ryukyu islands. Konchu 33:171-189.

10. Murray, M. G., and Thompson, W. F. 1980. Rapid isolation of high molecular weight plant DNA. Nucleic Acids Res. 8:4321-4325.

11. Notomi, T., Okayama, H., Masubuchi, H.,
Yonekawa, T., Watanabe, K., Amino, N., and Hase, T. 2000. Loop-medidated isothermal amplification of DNA. Nucleic Acids Res. 28:e63.

12. Planet, P., Jagoueix, S., Bove, J. M., and Garnier, M. 1995. Detection and characterization of the African citrus greening Liberobacter by amplification, cloning, and sequencing of the $r p l \mathrm{KAJL}-r p o \mathrm{BC}$ operon. Curr. Microbiol. 30:137-141.

13. Sanger, F., Nicklen, S., and Coulson, A. R. 1977. DNA sequencing with chain-terminating inhibitors. Proc. Natl. Acad. Sci. USA
74:5463-5467.

14. Subandiyah, S., Iwanami, T., Kondo, Y., Kobayashi, M., Tsuyumu, S., and Ieki, H. 2000. Comparison of $16 \mathrm{~S}$ rDNA and $16 \mathrm{~S} / 23 \mathrm{~S}$ intergenic region sequences among citrus greening organisms in Asia. Plant Dis. 84:15-18.

15. Villechanoux, S., Garnier, M., Laigret, F. Renaudin, J., and Bové, J. M. 1993. The genome of the non-cultured, bacterial-like organism associated with citrus greening disease contains the nusG-rplKAJL-rpoB gene cluster and the gene for a bacteriophage type DNA polymerase. Curr. Microbiol. 26:161-166. 